\title{
Les silences dans la dramaturgie giralducienne
}

Au théâtre, art de la parole autant que du jeu, le silence est avant tout absence de parole, pause dans l'échange des répliques, voire trou dans le dialogue ou même béance dans le discours d'un personnage : « [...] le silence est, au théâtre, une composante indispensable au jeu vocal et gestuel de l'acteur [...] ». (Pavis, $2004: 326$ ).

Contemporain de Craig qui prône un «théâtre du silence » qu'il considère comme devant être « le plus durable » (1964: 64), Giraudoux revendique son œuvre dramatique comme un théâtre littéraire, c'est-à-dire comme un théâtre de mots. Son théâtre recèle pourtant nombre de silences, indiqués ou non par des dispositifs techniques et rhétoriques. Quelle valeur peut-on leur attribuer? Quelle relation entretiennent-ils avec la parole et avec le jeu ? Certains silences ne jouent-ils pas un rôle rythmique, quasi musical, de respiration du texte ? Quels choix dramaturgiques induisent-ils ? S'agit-il d'une dramaturgie qui assume la mimesis ou qui, au contraire, la met en cause ?

Les dispositifs techniques et rhétoriques employés par Giraudoux pour indiquer les silences varient au cours de sa production dramatique : les didascalies explicites prescriptives émaillent sa première pièce et se font de plus en plus rares à mesure que l'on avance dans sa production dramatique. Siegfried comporte à plusieurs reprises l'indication «Un silence » (Giraudoux, $1982: 51,60,67,69,74,75)$, deux fois "Silence » (Giraudoux, $1982: 19$ ), une autre fois «Un temps» (Giraudoux, 1982 : 60). Faut-il les considérer comme interchangeables ? La mention « Silence» est associée à un silence partagé et lourd, comme en témoigne le qualificatif « angoissant » pour une des occurrences (Giraudoux, 1982: 19). Dans deux indications de jeu, nous trouvons l'adverbe "silencieusement» (Giraudoux, $1982: 14,34)$. Les autres didascalies sont dans Amphitryon 38, «Un silence » (Giraudoux, 1982:142), dans Électre «Silence» (Giraudoux, $1982: 669)$ et il faut attendre La Folle de Chaillot pour retrouver la mention «Un silence» (Giraudoux, $1982: 1021$ ).

Françoise Bombard - docteur ès lettres, a soutenu en 2009, à l'Université de Lyon, sa thèse sous le titre Les Objets dans le théâtre de Jean Giraudoux. Une esthétique du décalage et de la dissonance; elle en a fait paraître une version abrégée sous le titre Décalages et dissonances. Les objets dans le théâtre de Giraudoux (Cahiers Jean Giraudoux $\mathrm{n}^{\circ}$ 41, Presses Universitaires de Clermont-Ferrand, 2013). Elle a publié de nombreux articles sur cet auteur et d'autres (Bertrand, Crommelynck, Kacimi, Schéhadé). Adresse de correspondance : Grande Rue 01270 Coligny, France ; e-mail : francoise.bombard.sternli@orange.fr 
Or cet abandon des didascalies explicites va de pair avec un emploi de plus en plus fréquent des trois points de suspension dont certains, à l'évidence, correspondent à des silences. On peut penser que la complicité de l'auteur et de son metteur en scène, Louis Jouvet, explique en partie la disparition des marques textuelles explicites. Mais sans doute faut-il envisager d'autres hypothèses.

Par exemple, un locuteur attire l'attention de son interlocuteur sur un autre personnage ou sur un bruit par des verbes de perception. Cela nous vaut, sur le mode badin, une réplique du Droguiste d'Intermezzo à propos d'Isabelle et des petites filles :

Voyez-la à cheval sur ce baliveau, faisant valser cet ânon, en agitant un chardon, pendant que ses élèves dansent une ronde autour d'eux : la nécessité des ânons dans ce bas monde devient fulgurante... Celle des petites filles aussi, d'ailleurs... Regardez-les, Monsieur le Maire, les charmantes petites figures, les charmants petits dos... (Giraudoux, $1982: 283)$.

L’emploi du même procédé peut, au contraire, renforcer le tragique. Ainsi, lorsque Judith va proclamer la vérité de sa nuit avec Holopherne, elle dit à Suzanne : « [...]. Les Juifs sauront tout, Suzanne. [...] Écoute... Des gens approchent... C'est le châtiment qui arrive. » (Giraudoux, 1982 : 261). Plus sinistre encore est l'approche de la mort dans Fin de Siegfried: le recours à l'allégorie et les silences qui induisent une sorte de ralenti instaurent une atmosphère pesante :

Siegfried : Adieu, Geneviève. Toi, va à cette porte... On monte... Durand : Je n'entends rien. [...]. Siegfried : [...] Ouvre. Durand : Voilà... Siegfried : Non! Non! Ouvre vraiment. Pour celle-là, il faut ouvrir vraiment ! Durand : C'est ouvert. [...] Siegfried : Non, non, pour cette visite, il faut ouvrir la porte toute grande... [...]. Siegfried : Toute grande. L'instrument qu'elle porte est de travers. Elle ne peut passer... À deux battants... Silence. Vent léger. Siegfried : La voilà. Il ferme les yeux. [...]. Er ist gestorben! (Giraudoux, 1982 : 111).

Les trois points correspondent à la progression de l'agonie du blessé, mais c'est par l'indication "Silence. ", équivalente d'une ellipse à valeur euphémistique, que Giraudoux dit l'instant même de la mort, avant la constatation médicale en allemand dépourvue de toute émotion.

Parfois, les «trois points » amènent une phrase de commentaire du locuteur : soit sur son propre discours soit sur la situation. Ainsi, lors de la discussion entre la Folle de Chaillot et les petites gens, ses amis, qui veulent l'éclairer sur l'état de la société, la réplique du Chiffonnier comporte comme une parenthèse après les points de suspension, ce qui peut être joué comme un silence : «La Folle : je vous demande où vous voulez en venir ? [...]. Le Chiffonnier : À ceci, Comtesse... Tant pis ! Je lâche le morceau ! À ceci : le monde file un mauvais coton. " (Giraudoux, 1982 : 983). À l'acte I de Sodome et Gomorrhe, Dalila réservant les termes féminins pour désigner les qualités de Samson parle d'elle-même avec des mots de genre masculin : 
" [...] le charme, l'éclat et le prestige... Vous connaissez un mot masculin pour candeur ?... À part Dalila... Lia : Comment est-il ? Dalila : Le type que je déteste... Mais ce n'est pas un défaut. » (Giraudoux, $1982:$ 894). Le commentaire de Dalila suggère la perfidie légendaire du personnage. Le procédé est aussi employé à des fins comiques. Lorsqu'Agnès, dans L'Apollon de Bellac, met en pratique la leçon du monsieur de Bellac, à savoir dire aux hommes qu'ils sont beaux, les trois points encadrent la réaction amusée de son mentor qui s'est fait piéger : "Agnès : [...] Comment se fait-il que vous soyez si beau ? Le monsieur de Bellac : Mon père était très beau... Que je suis bête! Bravo, vous m’avez pris... » (Giraudoux, 1982 : 928).

Les " trois points " peuvent également indiquer un changement de sujet dans la réplique du locuteur. Au début de La Folle de Chaillot, il est dicté par les circonstances. Le Prospecteur (un de ceux qui veulent explorer le sous-sol parisien pour exploiter le pétrole) prend la parole, mais son discours est aussitôt perturbé par l'entrée en scène d'un personnage extravagant : « Le Prospecteur : Voici mon plan... Mon Dieu, quelle est cette figure ?" (Giraudoux, 1982 : 964). La didascalie descriptive de la Folle de Chaillot n'intervenant qu'après dans le texte, cela peut suggérer un jeu de scène : la Folle serait d'abord invisible du public. Giraudoux emploie le même procédé un peu plus tard pour une entrée en scène qui bouleverse encore plus les desseins du Prospecteur : "Parfait. Midi sonne... Mais qu'arrive-t-il ? (Le sauveteur du pont de l'Alma entre portant un corps). Le Prospecteur : C'est Pierre!» (Giraudoux, 1982 : 969). En effet, à midi, le pavillon de l'ingénieur - opposé aux manœuvres du Prospecteur - devait sauter, dynamité par Pierre, mais nous apprenons ensuite que ce dernier s'est jeté dans la Seine au lieu d'agir.

Dans Sodome et Gomorrhe et Pour Lucrèce, pièces où abondent les dialogues par tirades qui se répondent en duos, en trios ou en quatuors - Sodome et Gomorrhe a été présentée par Hébertot comme un " oratorio " (Giraudoux, 1982 : 1672) -, les " trois points " ne marquent ni une hésitation ni un temps de réflexion du personnage. Il semble bien qu'ils aient un rôle rythmique de respiration du texte. Pour Lucrèce en comporte plusieurs exemples. Ainsi du rythme haché qu'impose la situation lorsqu'Armand revient après avoir tué Marcellus en duel : " Il meurt... j'ai pu échapper à la police... j'ai voulu vous revoir avant d'être rejoint... » (Giraudoux, 1982 : 1099). À l'acte III, une réplique de Lucile exigeant de Paola qu'elle se mette à genoux et demande pardon est ainsi construite sur un rythme descendant, ascendant, descendant ponctué par les silences : «À Marcellus... À nos maris... À Barbette... À tous les vivants et les morts... À moi... À vous... » (Giraudoux, 1982 : 1111). La valeur rythmique des silences correspond ici à la respiration de l'acteur qui interprétera le personnage. Le procédé est le même pour le début d'une tirade du Procureur marqué par des rythmes binaires mais on sent dans cette envolée rhétorique le personnage rompu à la pratique des réquisitoires et des discours officiels : « Pardon, Lucile... Et merci. Non seulement pour moi qui ne vais plus traîner une vie misérable, qui sors ennobli de ce cauchemar... Merci pour la ville infâme, pour le monde à qui tu infliges cette leçon... [...].»(Giraudoux, 1982 : 1114). 
Les « trois points » introduisent parfois un jeu impliqué par la réplique à défaut de didascalie. Tel est le cas, dans La Guerre de Troie n'aura pas lieu, de la séquence de la «photographie » d'Hélène par Demokos qui prétend fixer son image (mais tout ceci n'est affaire que de mots) : «Demokos : [...]. J'ai dans la main un magnifique oiseau que je vais lâcher... Là, tu y es ?... C'est cela... Arrange tes cheveux et souris un beau sourire. [...]. Ne bouge plus... Une ! Deux ! Trois ! Voilà... c'est fait, tu peux partir... » (Giraudoux, $1982: 515)$.

En l'absence de tout dispositif technique ou rhétorique, le jeu muet peut instaurer un silence qui sape le dialogue entre les personnages. Amphitryon 38 offre un exemple intéressant de ce procédé, les didascalies contribuant à démentir le discours sur la paix et à instaurer un climat angoissant auquel les deux personnages parlants restent étrangers : "Dans le dos même de Sosie, gravissant degré par degré l'escalier qui mène à la terrasse, surgit et grandit un guerrier géant, en armes ". À mesure que Sosie expose sa proclamation aux Thébains, la menace progresse comme un démenti ironique aux paroles glorifiant la paix : "Le guerrier gravit les derniers degrés et se rapproche. [...]. Le guerrier est derrière Sosie. Le Guerrier : Tu dis ? " (Giraudoux, $1982: 121-122)$.

La progression silencieuse du guerrier géant ne rencontre-t-elle pas le " théâtre du silence » de Craig qui accorde la primauté au visuel, parce qu'il parle davantage à l'imagination que les mots ? Bien sûr, quoique le silence puisse miner le pouvoir des mots, Giraudoux est loin de la radicalité de Craig qui vise l'éviction du mot : du silence giralducien surgit brutalement la parole belliqueuse qui finit par déferler en tirades.

Quoique Giraudoux rejette le naturalisme, il subsiste dans sa première pièce un usage des silences qui relève d'une dramaturgie assumant la mimesis dans la mesure où elle reste tributaire de la psychologie. Or, selon Pavis :

Dans le théâtre réaliste [...] les silences sont laissés à la libre interprétation du comédien [qui] les réalise (en accord avec le metteur en scène) selon l'analyse psychologique de son personnage [...]. La gestualité et la mimique subviennent alors aux besoins de ces vides, et les silences ne sont que l'envers et la préparation de la parole [...]. (2004:326).

Certains silences sont chargés d'une grande tension dramatique : c'est particulièrement vrai dans Siegfried. La rencontre entre Robineau - venu à Gotha à la demande de son ancien ami allemand Zelten - et ce dernier, est d'abord tendue : ils se revoient après la guerre. La didascalie liminaire de la scène propose un jeu muet qui souligne à la fois l'émotion de ces retrouvailles, l'embarras et la difficulté à renouer le dialogue interrompu : "Ils restent à distance, se contemplant silencieusement à travers toute la scène. Zelten : Voilà ! Robineau : Voilà ! [...] " (Giraudoux, 1982 : 14). Pour dissiper la gêne, ils s'identifient mutuellement puis font assaut d'épithètes homériques avant d'échanger des banalités, toutes ces paroles cherchant à remplir, coûte que coûte, le silence lourd de tout ce qui les a séparés : " (Il se rapproche. Affectant la conversation 
familière.) Bonjour, Robineau ! Robineau : Bonjour, Zelten. Zelten : Tu vas bien ? Robineau : Pas mal, et toi ? Un silence. » (Giraudoux, 1982:15).

Geneviève et Robineau, les deux Français mandés à Gotha par Zelten, éprouvent un malaise croissant lors du dialogue qui porte sur Jacques Forestier, le fiancé disparu de Geneviève. Après les insinuations de Zelten - «Il n'est pas mort ! Il n'est que disparu ! [...] Il est disparu, il peut reparaitre. » - la tension ne fait que s'amplifier : "L'agitation de Robineau s'accroit. Silence angoissant pendant lequel on entend une porte s'ouvrir sur le palier d'en haut. » (Giraudoux, $1982: 19$ ). La qualification du silence lui donne une dimension psychologique proche de ce qu'on pourrait lire dans un roman de la part d'un narrateur omniscient, mais comment rendre palpable ce « silence angoissant » lors d'une représentation ? La simultanéité entre le silence partagé par deux personnages et l'émergence d'un bruit contribue à renforcer le poids de ce silence. À partir de là, comme dans un mélodrame, les silences, la voix de Siegfried en off, le bruit de ses pas prennent une importance telle qu'ils étouffent le dialogue réduit à des échanges de plus en plus brefs :

On entend la voix de Siegfried. Zelten : Écoutez! Geneviève : Quoi ? Que voulez-vous dire? Mais c'est la voix de Jacques !... (En haut la voix se tait). C’était la voix de qui ? Zelten : [...]. Du conseiller Siegfried. Geneviève, allant vers l'escalier et criant : Jacques !... Silence. (Giraudoux, 1982 : 19).

Notons que Giraudoux emploie une curieuse formulation (« la voix se tait») pour indiquer l'arrêt du son venant du hors scène, comme si le mot silence devait être réservé à ce qui se passe en scène.

Le jeu sur les silences est un moyen efficace de renforcer la valeur dramatique de la première confrontation entre Geneviève et Siegfried. L'incapacité de la jeune femme à parler, tant son émotion est forte, est soulignée par un jeu de mimiques qui ponctue les répliques du héros :

Siegfried, se présentant: Geheimrat Siegfried. Geneviève incline la tête. Siegfried : Je vous croyais une vieille, très vieille dame. Je n'ose plus dire mon projet. Geneviève le regarde toujours. Je ne me trompe pas ?... Vous êtes cette dame canadienne française, qu'on vient de m'annoncer? Geneviève hoche affirmativement la tête. Vous me comprenez bien? [...] Me rendriez-vous ce service de venir à ce moment ? Dès demain ? Robineau : Accepte. Geneviève incline la tête. (Giraudoux, $1982: 20$ ).

À l'acte III, après la révélation de son identité véritable dans la scène précédente - «Geneviève : [...] tu es Français, tu es mon fiancé, Jacques, c'est toi. » (Giraudoux, 1982 : 54) -, Siegfried est incapable de renouer le dialogue avec Eva (celle qui l'a recueilli blessé et a fait de lui un homme d'État allemand) :

Eva : Siegfried! Siegfried tourne la tête vers elle. Eva : C'est moi, Siegfried... Siegfried : Geste de lassitude. Eva : Si c'est un crime d'avoir partagé avec toi ma patrie, pardon, 
Siegfried. Siegfried : Geste vague. Eva : Si c'est un crime [...] pardon. Siegfried : Cela va... Laisse-moi. (Giraudoux, $1982: 54$ ).

Dans les actes III et IV, les dialogues entre Geneviève et le héros sont lourds de silences, en particulier dans le grand duo d'amour de l'acte IV. Le silence partagé est alors le lieu d'une communication muette, d'une ré-union des deux protagonistes :

Geneviève : Et les montagnes ! [...]. Tu arrives à leur sommet avec des sacs à main, des ombrelles, et moi presque nue. Un silence. [...] Siegfried : Où vous-ai-je rencontrée ? Geneviève : Au coin d'une rue, près d'un fleuve. [...] Tu sais être heureux. Un silence. Siegfried : Je vous aimais ? Geneviève : Toi seul l'a su. Je comptais sur ton retour pour le savoir moi-même. Un silence. Siegfried : Nous étions seulement fiancés, Geneviève ? [...]. Geneviève : Non, amants. (Un silence). Tu sais être cruel. [...]. Tu me parlais de mon passé à moi. Tu en étais jaloux. Tu ne me croyais pas. J’étais le Forestier d'alors. Un silence. Siegfried, qui tient toujours Geneviève : Qui es-tu, Geneviève? (Giraudoux, 1982 : 74-75).

Chaque réplique de la jeune femme reconstruit le passé de Jacques, leur passé, et les silences qui ponctuent le dialogue expriment le chemin que font ces révélations dans l'esprit et le cœur de Siegfried qui peu à peu retrouve son identité première. On voit ici que Giraudoux sait faire de la «non-présence » de la parole la plus haute expression de l'intériorité. Dans le texte primitif de Sodome et Gomorrhe n'avait-il pas ajouté à une tirade de Jean énonçant les qualités de l'homme : «Et il sait se taire de ce silence qui est le clavier ouvert aux voix intérieures » (Giraudoux, 1982:1696) ?

Le silence, non content d'être exploité pour sa qualité affective, est thématisé par Giraudoux qui le charge du non-dit ou de l'indicible, et va jusqu'à le mettre en scène.

Nous ne parlons que par la différence qui nous tient à distance de la parole, seulement parce que nous parlons et toutefois pas encore : ce " pas encore » ne renvoie pas à une parole idéale, au verbe supérieur dont nos paroles humaines seraient l'imparfaite imitation mais il constitue la décision même de la parole, dans sa non-présence, cet à venir qu'est toute parole tenue pour présente, d'autant plus insistante qu'elle désigne et engage le futur, qui est aussi un futur à parler, cette non-parole qui appartient au langage et qui pourtant, chaque fois que nous parlons essentiellement, nous met hors du langage de même que nous ne sommes jamais plus près de parler que dans la parole qui nous en détourne. (Blanchot, $1969: 44-45)$.

Certains silences sont, en effet, moins chargés d'émotion, partagée ou non, que lourds d'un sens qu'il s'agit d'interpréter.

Soulevant la question du rapport du dire et du taire, le non-dit que suggèrent des silences marqués par les " trois points » est de l'ordre de l'indicible. Lucile accepte des mains de Paola le mouchoir de Marcellus qui pourtant avait été le faux indice du viol simulé, mis en scène par Paola : faut-il y voir l'aveu inconscient du désir ? 
« Paola : [...]. Il vous manque de cette nuit un souvenir palpable. [...]. Je le tenais de Marcellus. Lucile : Bien, donnez... » (Giraudoux, 1982 : 1107). À la fin de la pièce, Lucile, qui a absorbé du poison, répond à la fureur et au mépris de son mari qui la rejette, la croyant coupable d'adultère, par deux euphémismes qui masquent son suicide : «Le Procureur : [...]. Partez, Lucile, quittez cette maison. Lucile : Rassurezvous. Je vais partir... [...]. Voilà. C’est fini...» (Giraudoux, 1982 : 1109-1110).

Le théâtre de Tchekhov nous a habitués aux silences qui correspondent à « la parole réprimée » des personnages (Pavis, 2004 : 326), et, parfois, Giraudoux paraît s'en souvenir. Quand, au début de Judith, le rabbin Joachim demande à Jean, capitaine de l'armée juive, de dire la vérité sur la situation de Béthulie assiégée, l’officier peine à sortir du silence, et ce silence est un aveu. Judith, qui s'enferme d'abord dans le déni de la réalité, parle tandis que Jean se tait : « Joachim [...]. Réponds... Judith : Mais réponds donc! Par un mot! Par une phrase ! Jean : Tu es cruelle ! Judith : [...] À ton visage seul, ta phrase se devine... Jean : J'en remercie Dieu... Judith : Tu le remercies aussi dêtre vaincu? " (Giraudoux, $1982: 211$ ).

Cette idée que le silence peut « faire signe », voire être un signe, nous la retrouvons dans Pour Lucrèce, lorsque Lucile persiste dans son mutisme, quoique Armand fasse pour obtenir d'elle une parole : "Armand : [...] dites à votre amie que je la conjure de quitter son silence. Si elle ne me parle pas, elle me fonde à croire que je suis un mari volage ou un mari trompé [...]. » (Giraudoux, 1982 : 1045). Et Eugénie, l’amie de Lucile qui essaie de sauver la situation, commente : «Si Lucile vous cédait maintenant, c'est qu'elle vous jugerait stupide, ce serait pour vous dire que vous êtes comme les autres [...] sans intelligence pour le cœur et le silence. » (Giraudoux, 1982 : 1046).

Mais interpréter un silence n'est pas sans risque, comme en témoigne Amphitryon 38. Une fois que Léda est entrée dans la chambre conjugale pour éviter à Alcmène la visite de Jupiter, Amphitryon se méprend sur la nature du silence de son épouse : « [...] Je peux venir, Alcmène ?... Elle ne répond pas : je la connais, c'est qu'elle est prête... Quelle délicatesse, c'est par son silence qu'elle me fait signe, et quel silence ! Comme il résonne ! Comme elle m'appelle ! [...]» (Giraudoux, 1982 : 173). Cette réplique est déjà une forme de mise en abyme du silence.

Il en est d'autres. Dans Siegfried, l'absurdité apparente de la situation (une supposée institutrice canadienne censée donner des leçons de français et restant enfermée dans le silence) est soulignée par le héros : "J'espère que ce n'est pas avec une dame muette que je vais prendre mes leçons? " (Giraudoux, $1982: 20$ ). Et Geneviève ne sort de son mutisme qu'à la question : «Et le silence, Mademoiselle, comment ditesvous cela au Canada ?». Giraudoux nous fait alors passer de l'action scénique à un échange sur les langues : "Geneviève, lentement, comme en rêve : Et en allemand ? Siegfried : Stille! Silentium ! Geneviève : Cela se dit silence.» (Giraudoux, 1982 : 21).

La mise en abyme va quelquefois de pair avec la «surthéâtralisation », c'est-à-dire " un degré plus élevé [de la théâtralisation qui] consiste à accentuer le côté théâtral, en montrer les ficelles, le faire se dédoubler comme dans un miroir. » (Kowzan, 1983 : 100). Dans Amphitryon 38, il s'agit pour Mercure - qui seconde Jupiter dans 
ses entreprises de séduction et qui endosse ici le rôle de meneur de jeu - de prendre le temps de regarder et d'évaluer les étapes de la métamorphose du dieu en homme, et surtout d'en critiquer les insuffisances :

Mercure : C'est votre corps entier qui doit être sans défaut... Venez là, à la lumière, que je vous ajuste votre uniforme d'homme... Plus près, je vois mal... Jupiter : Mes yeux sont bien ? Mercure : Voyons vos yeux... Trop brillants... Ils ne sont qu'un iris [...]. (Giraudoux, $1982: 133)$.

$\mathrm{Au}$ dénouement, alors que Mercure convoque tous les personnages ainsi que " chants, musique et foudre ", Jupiter cède à la prière d'Alcmène, Giraudoux jouant sur le silence associé à la vie privée du couple et sur celui du public : "Alors évidemment Mercure se trompe! Alors c'est l'aparté des apartés, le silence des silences. [...]. Vous tous spectateurs, retirez-vous sans mot dire en affectant la plus complète indifférence. » (Giraudoux, 1982 : 195). Le jardinier d'Électre, à la fin de son monologue du Lamento, va jusqu’à nous faire écouter le silence :

[...] Moi, ç’a toujours été les silences qui me convainquent... [...] je vous conjure, Dieu, comme preuve de votre affection, de votre voix, de vos cris, de faire un silence, une seconde de votre silence... C’est tellement plus probant. Écoutez... Merci. (Giraudoux, 1982 : 643).

L'adresse aux spectateurs, là encore, rompt avec les conventions : réclamer le silence (et non les applaudissements) est une façon de suspendre le temps et d'établir une autre relation du public au spectacle auquel il assiste.

Le Trompette, quant à lui, musicalise le silence : «[...] si je suis célèbre parmi les trompettes à une note, c'est qu'avant de sonner, ma trompette à la bouche, j'imagine d'abord tout un développement musical et silencieux, dont ma note devient la conclusion. » (Giraudoux, 1982 : 120). Comment mieux dire que la musique, comme la parole, naît du silence, dans lequel elles trouvent l'une et l'autre leur force et peut-être leur suprême accomplissement?

Les silences qui correspondent à l'expression d'une émotion, soit par la communication muette soit au contraire par la difficulté à parler, sont à l'image de ce qui se passe dans une conversation. Il en va de même pour ceux qui mettent en valeur un élément du discours ou de la réalité sur lequel le personnage locuteur cherche à attirer l'attention. Par ailleurs, les silences qui suggèrent le rythme de la réplique doivent être mis en relation avec l'importance accordée par Giraudoux à la respiration et au corps de l'acteur. Dans tous ces cas de figures, les silences relèvent d'une dramaturgie de la mimesis : le théâtre comme reflet de la vie. À l'inverse, certains silences induisent une rupture qui peut être le fait de la "surthéâtralisation » ou de la mise en abyme de la notion même de silence. D'autres minent le discours et s'avèrent une mise en cause de la capacité du langage à tout exprimer : ils sont comme la limite assignée au pouvoir des mots. 


\section{BIBLIOGRAPHIE}

Abiteboul M. 2011. La parole, le silence, le cri au théâtre. Théâtre du monde. 11.

Blanchot M. 1969. L'Entretien infini. Paris. Gallimard.

Graig E. G. 1964. Le Théâtre en marche. Traduction M. Beerblock. Paris Gallimard. Pratique du théâtre.

Giraudoux J. 1982. Théâtre complet. Paris. Gallimard. coll. «Bibliothèque de la Pléiade ».

Kowzan T. 1983. Vers la surthéâtralisation dans l'œuvre dramatique de Jean Giraudoux. Cahiers Jean Giraudoux 12, 100-112.

Pavis P. 2004. Dictionnaire du théâtre. Paris. Armand Colin.

\section{The silences in Giraudoux' dramaturgy}

ABSTRACT: Technical and rhetorical devices used by Giraudoux to indicate silence vary during its dramatic production. Silences have to do with relationships of the characters, both emotionally and dialogal, and are sometimes loaded the unspoken or unspeakable. By their relationship to the word and to the game, they are at the heart of a dramaturgy that oscillates between mimesis and rupture. And certain silences are like the limit assigned to the power of words in a theater yet claimed as literary.

Keywords: game, mimesis, silence, unsaid, unspeakable. 